\title{
WHETHER TO SELL OR HOLD A STOCK
}

\author{
RAYMOND RISHEL*
}

\begin{abstract}
For a simple model of a stock, whose stock price is a geometric Brownian motion in which the drift rate changes back and forth between positive and negative values, optimal selling times are computed. An infinite optimal selling time meaning you should just hold the stock.
\end{abstract}

Key words: stock selling times, optimal stopping, buy and hold, variational inequalities

1. Introduction. Motley Fool advises individual investors to use the "buy and hold" strategy, that is to buy "good stocks" which "will grow" and hold them. What is a "good stock"? All stocks have periods of growth and periods of decline. If an investor's stock price declines, should he sell the stock? Will it recover and regain it's value? If so, he should hold it, if not he should sell it.

These problems are analyzed for a simple stock price model. The model for the stock price is given by a geometric Brownian motion whose drift rate is a two state jump Markov process. One state of the drift rate is positive. In this state the stock price should increase. The other state of the drift rate is negative. In this state the stock price should decrease. The randomness of the Brownian motion makes these periods of increase or decrease only approximate.

For this model whether a stock is "good" or not depends on the solution of a differential equation. This solution is either positive on $[0,1]$ or there is an $x^{*}$ such that it is positive on $\left(x^{*}, 1\right]$ and negative on $\left[0, x^{*}\right)$. When the solution is positive the stock should not be sold. When it is not, the stock should be sold as follows. Let $x(t)$ denote, the conditional probability that the drift rate is in the increasing state, given observations of the stock price up to time t. The stock should be sold at the first time $x(t)$ hits $\left[0, x^{*}\right]$. This selling time has finite expected value, so the stock will always be sold after a finite time in this case.

Optimal stock selling problems for a model of this type have been treated by Q. Zhang in [9]. In [9], the stock selling times are first times the stock price leaves an interval. In the current paper, stock selling times are stopping times depending on past stock prices. These include the selling times of [9]. It appears that the methods, and even the results, of the two papers are quite different. In [7] Rishel and Helmes computed optimal selling times for a failing stock whose drift rate jumped once from an increasing rate to a decreasing rate. In their study [2],[3] Blanchet-Scallet, Diop, Gibson, Talay, and Tanre compare technical analysis techniques against using an

*Department of Mathematics, University of Kentucky, Lexington Kentucky 40506, E-mail: rishel@ms.uky.edu 
optimal portfolio policy but with misspecified coefficients for a one jump model of the type above.

2. The stock price model. Consider a stock whose price satisfies the stochastic differential equation and initial condition

$$
d S(t)=S(t)(a(t) d t+\sigma d w(t)), \quad S(0)=S .
$$

In (2.1) $S$ and $\sigma$ are constants, $w(t)$ is a standard Brownian motion process, and $a(t)$ is a jump Markov process. The jump Markov process $a(t)$ has two states $a$ and $b$ with $a>0$ and

$b<0$. The generator of the process is given by

$$
\left(\begin{array}{cc}
-c & k \\
c & -k
\end{array}\right) .
$$

The constant a gives the rate of increase, $b$ the rate of decrease, $1 / c$ is the expected time the drift $a(t)$ stays in state $a, 1 / k$ is the expected time the drift $a(t)$ stays in state $b$.

The investor watches the stock prices $S(t)$ and makes decisions based on these. Thus let

$$
F_{t}=\sigma[S(r), 0 \leq r \leq t]
$$

be the sigma field generated by his observations up to time $t$, and his selling time $\tau$ be a $F_{t}$ adapted stopping time.

We could consider the problem of choosing a selling time $\tau$ to maximize the expected logarithmic utility

$$
E[\ln (S(\tau))]
$$

However, we wish to consider infinite selling times, and the criterion (2.4) is not well defined for infinite selling times $\tau$. Instead we will consider maximizing

$$
\liminf _{t \rightarrow \infty} E[\ln (S(\tau \wedge t))]
$$

Since the investor observes the stock price $\mathrm{S}(\mathrm{t})$, but cannot observe the growth rate $\mathrm{a}(\mathrm{t})$, this is a partially observed optimal stopping problem. A technique using nonlinear filtering formulas to reduce this type of partially observed problem to a completely observed problem has become standard. This type of technique was first used by Shiryayev [8] . 
3. An Equivalent Completely Observed Problem. The solution of the stock price equation (2.1) is given by

$$
S(t)=S e^{\int_{0}^{t} a(u) d u+\sigma w(t)-1 / 2 \sigma^{2} t}
$$

This may be checked using Ito's differential rule. Thus

$$
\ln (S(t))-\ln S+1 / 2 \sigma^{2} t=\int_{0}^{t} a(u) d u+\sigma w(t) .
$$

Now (3.2) implies observing $\mathrm{S}(\mathrm{t})$ is equivalent to observing $\mathrm{y}(\mathrm{t})$ where

$$
y(t)=\int_{0}^{t} a(u) d u+\sigma w(t)
$$

and the sigma fields

$$
\sigma[S(r), 0 \leq r \leq t] \text { and } \sigma[y(r), 0 \leq r \leq t]
$$

are equal.

Nonlinear filtering results, for instance, Davis, Markus [4], Lipster, Shiryayev [6], govern conditional probabilities of states of a jump Markov process given measurements of the type (3.3). In particular, if

$$
x(t)=\operatorname{Pr}[a(t)=a \mid y(r), 0 \leq r \leq t] \text { and } x_{0}=\operatorname{Pr}[a(0)=a]
$$

then $\mathrm{x}(\mathrm{t})$ is a solution of

$$
d x(t)=\{-c x(t)+k(1-x(t))\} d t+\left(\frac{a-b}{\sigma}\right)(1-x(t)) x(t) d \nu(t), \quad x(0)=x_{0}
$$

In (3.6) $\nu(t)$ is a Brownian motion process called the innovations process. It is related to the Brownian motion process $w(t)$ through the relationship

$$
\int_{0}^{t} a(u) d u+\sigma w(t)=\int_{0}^{t}\{a x(u)+b(1-x(u))\} d u+\sigma \nu(t) .
$$

The relationship (3.7) allows the stock price equation (2.1) to be rewritten as

$$
d S(t)=S(t)\{(a x(t)+b(1-x(t))) d t+\sigma d \nu(t)\}, \quad S(0)=S .
$$

Applying Ito's differential rule to $\ln (S(t))$ using (3.8) gives

$$
d \ln (S(t))=\left(a x(t)+b(1-x(t))-\frac{1}{2} \sigma^{2}\right) d t+\sigma d \nu(t), \quad \ln S(0)=\ln S .
$$

In integrated form $(3.9)$ is

$$
\ln (S(t))=\ln S+\int_{0}^{t}\left(a x(u)+b(1-x(u))-\frac{1}{2} \sigma^{2}\right) d u+\int_{0}^{t} \sigma d \nu(u)
$$


Formula (3.10) also holds with $t$ replaced by the random time $t \wedge \tau$. Doing this and taking expectations gives

$$
E[\ln (S(t \wedge \tau))]=\ln S+E\left[\int_{0}^{t \wedge \tau}\left(a x(u)+b(1-x(u))-\frac{1}{2} \sigma^{2}\right) d u\right]
$$

The expected value of the stochastic integral

$$
E\left[\int_{0}^{t \wedge \tau} \sigma d \nu(u)\right]
$$

is zero since the upper limit has finite expected value.

The argument above shows that our original problem of finding a selling time to maximize (2.5) subject to (2.1) holding is equivalent to the problem.

Maximize

$$
\liminf _{t \rightarrow \infty} E\left[\int_{0}^{t \wedge \tau}\left(a x(u)+b(1-x(u))-\frac{1}{2} \sigma^{2}\right) d u\right]
$$

where $\mathrm{x}(\mathrm{t})$ is a solution of

$$
d x(t)=(-c x(t)+k(1-x(t))) d t+\left(\frac{a-b}{\sigma}\right)(1-x(t)) x(t) d \nu(t), \quad x(0)=x_{0}
$$

Since $\mathrm{x}(\mathrm{t})$ was defined by (3.5) and (3.4) holds, $\mathrm{x}(\mathrm{t})$ is observable.

4. Preliminary Considerations. Notice if $\sigma^{2} \geq 2 a$, that the criteria (3.13) is nonpositive. Thus in this case, it is optimal to sell the stock immediately. It could be said the stock is too risky in this case. In the remainder of the paper we shall assume $\sigma^{2}<2 a$.

We shall need the following properties of $x(t)$. It follows from Theorem 2 p.149 of [5], that for $t>0$, the solution of (3.14) satisfies,

$$
\operatorname{Pr}[0<x(t)<1]=1 .
$$

Lemma 1. For $0<y<1$ define

$$
T(y)=\text { first time } x(t) \text { hits }[0, y]
$$

then

$$
E[T(y)]<\infty .
$$

Lemma 1 can be proved by an argument similar to Theorem [2] p. 108 of [5]. From (4.1) it follows that

$$
\lim _{y \rightarrow 0} T(y)=+\infty
$$


5. Variational Inequality Optimality Conditions. Variational inequalities for solving optimal stopping problems were introduced by Bensoussan and Lions and are discussed in [1]. Consider the operator

$A[V](x)=a x+b(1-x)-\frac{\sigma^{2}}{2}+(-c x+k(1-x)) V^{\prime}(x)+\frac{1}{2}\left(\frac{a-b}{\sigma}\right)^{2}(1-x)^{2} x^{2} V^{\prime \prime}(x)$

and the variational inequality

$$
A[V](x) \leq 0, \quad V(x) \geq 0, \quad V(x) A[V](x)=0 .
$$

A solution $r(x)$ of the differential equation

$$
a x+b(1-x)-\frac{\sigma^{2}}{2}+(-c x+k(1-x)) r(x)+\frac{1}{2}\left(\frac{a-b}{\sigma}\right)^{2}(1-x)^{2} x^{2} r^{\prime}(x)=0
$$

is given by

$$
r(x)=C(x)^{-1} \int_{x}^{1} B(y) C(y) d y
$$

where

$$
\begin{gathered}
B(x)=\frac{a x+b(1-x)-\frac{\sigma^{2}}{2}}{\frac{1}{2}\left(\frac{a-b}{\sigma}\right)^{2}(1-x)^{2} x^{2}}, \\
C(x)=\left(\frac{1-x}{x}\right)^{2\left(\frac{\sigma}{a-b}\right)^{2}(c-k)} e^{-\left(\frac{2 \sigma^{2}}{(a-b)^{2}}\right)\left(\frac{c}{1-x}+\frac{k}{x}\right)} .
\end{gathered}
$$

On $[0,1], C(x)$ is nonnegative and $B(x)$ changes sign at most once. Thus $r(x)$ changes sign at most once on $[0,1]$. A L'Hospital's rule argument shows

$$
\lim _{x \rightarrow 1} r(x)=\frac{2 a-\sigma^{2}}{2 c} .
$$

Lemma 2. If there is an $x^{*}$ in $(0,1)$ for which $r\left(x^{*}\right)=0$, then

$$
V(x)=\left(\begin{array}{c}
\int_{x^{*}}^{x} r(u) d u \text { if } x^{*} \leq x \leq 1 \\
0 \text { if } 0 \leq x \leq x^{*}
\end{array}\right)
$$

is a solution of the variational inequality (5.2).

Proof. Since $2 a>\sigma^{2},(5.7)$ implies $r(x)$ is positive near $x=1$. Since $r(x)$ changes sign only once and does so at $x^{*}, r(x)$ is positive on $\left(x^{*}, 1\right]$. Thus $V(x) \geq 0$. 
From (5.1), (5.3) and ( 5.8),

$$
A[V](x)=\left(\begin{array}{c}
0 \text { if } x^{*} \leq x \leq 1 \\
a x+b(1-x)-\frac{\sigma^{2}}{2} \text { if } 0 \leq x \leq x^{*}
\end{array}\right) .
$$

Since $B(x)>0$ if $a x+b(1-x)-\frac{\sigma^{2}}{2}>0,(5.4),(5.5)$, and (5.6) imply that

$$
x^{*}<\frac{\frac{\sigma^{2}}{2}-b}{a-b} \text {. }
$$

This implies

$$
a x+b(1-x)-\frac{\sigma^{2}}{2}<0 \text { if } 0<x \leq x^{*} .
$$

giving that $A[V](x) \leq 0$.

That $V(x) A[V](x)=0$ follows from (5.8) and (5.9).

THEOREM 1. If there is an $x^{*}$ in $(0,1)$ for which $r\left(x^{*}\right)=0$ then, $T\left(x^{*}\right)=$ first time $x(t)$ hits $\left[0, x^{*}\right]$, is the optimal selling time for the problem (3.13), (3.14).

Proof. Notice that $V(x)$ given by (5.8) is continuously differentiable and twice continuously differentiable except at the point $x^{*}$. Thus Ito's differential rule holds for $V(x)$, and gives

$$
\begin{aligned}
V(x(t))=V( & x)+\int_{0}^{t}\left\{[-c x(u)+k(1-x(u))] V^{\prime}(x(u))\right. \\
+ & \left.\frac{1}{2}\left(\frac{a-b}{\sigma}\right)^{2}(1-x(u))^{2} x(u)^{2} V^{\prime \prime}(x(u))\right\} d u \\
& +\int_{0}^{t}\left(\frac{a-b}{\sigma}\right)(1-x(u)) x(u) V^{\prime}(x(u)) d \nu(u)
\end{aligned}
$$

Now

$A[V](x)-\left(a x+b(1-x)-\frac{\sigma^{2}}{2}\right)=(-c x+k(1-x)) V^{\prime}(x)+\frac{1}{2}\left(\frac{a-b}{\sigma}\right)^{2}(1-x)^{2} x^{2} V^{\prime \prime}(x)$.

So (5.12) can be rewritten as

$$
\begin{aligned}
V(x(t))= & V(x)+\int_{0}^{t}\left\{A[V](x(u))-\left(a x(u)+b(1-x(u))-\frac{\sigma^{2}}{2}\right)\right\} d u \\
& \left.+\int_{0}^{t}\left(\frac{a-b}{\sigma}\right)(1-x(u)) x(u) V^{\prime}(x(u))\right\} d \nu(u)
\end{aligned}
$$

For a selling time $\tau,(5,14)$ holds with $t$ replaced by the random time $\tau \wedge t$. Doing this and taking expectations gives

$$
E[V(x(\tau \wedge t))]=V(x)+E\left[\int_{0}^{\tau \wedge t} A[V](x(u)) d u-E\left[\int_{0}^{\tau \wedge t}\left(a x(u)+b(1-x(u))-\frac{\sigma^{2}}{2}\right) d u\right] .\right.
$$


Since its integrand is bounded and its upper limit is finite, the expected value of the stochastic integral in obtaining (5.15) was zero. Rearranging (5.15)

$V(x)-E\left[\int_{0}^{\tau \wedge t}\left(a x(u)+b(1-x(u))-\frac{\sigma^{2}}{2}\right) d u\right]=E[V(x(\tau \wedge t))]-E\left[\int_{0}^{\tau \wedge t} A[V](x(u)) d u\right]$.

Thus since the variational inequality asserts the two terms on the right hand side of (5.15) are nonnegative,

$$
V(x) \geq E\left[\int_{0}^{\tau \wedge t}\left(a x(u)+b(1-x(u))-\frac{\sigma^{2}}{2}\right) d u\right] .
$$

Replacing $\tau$ by $T\left(x^{*}\right)$ in (5.16) we obtain

$$
V(x)-E\left[\int_{0}^{T\left(x^{*}\right) \wedge t}\left(a x(u)+b(1-x(u))-\frac{\sigma^{2}}{2}\right) d u\right]=E\left[V\left(x\left(T\left(x^{*}\right) \wedge t\right)\right)\right] .
$$

The last term in (5.16) is zero for $\tau=T\left(x^{*}\right)$ because, either $T\left(x^{*}\right)=0$, or $x(u)$ is in $\left[x^{*}, 1\right]$, on which $A[V](x)=0$. Since the following all hold: $V(x)$ is bounded, $V(x)$ and $x(t)$ are continuous, $x\left(T\left(x^{*}\right)\right)=x^{*}, \quad V\left(x^{*}\right)=0$, and

$$
\lim _{t \rightarrow \infty} T\left(x^{*}\right) \wedge t=T\left(x^{*}\right),
$$

the dominated convergence theorem implies

$$
\lim _{t \rightarrow \infty} E\left[V\left(x\left(T\left(x^{*}\right) \wedge t\right)\right)\right]=0
$$

Thus

$$
V(x)=\lim _{t \rightarrow \infty} E\left[\int_{0}^{T\left(x^{*}\right) \wedge t}\left(a x(u)+b(1-x(u))-\frac{\sigma^{2}}{2}\right) d u\right]
$$

and (5.17) and (5.21) imply $T\left(x^{*}\right)$ is the optimal selling time.

THEOREM 2. If $r(x)$ is positive on $[0,1]$ the stock should not be sold.

Proof. Let

$$
z(x)=-\int_{x}^{1} r(z) d z .
$$

Ito's differential rule, (3.14), and (5.22) imply

$$
\begin{aligned}
z(x(t))=z\left(x_{0}\right) & +\int_{0}^{t}\{(-c x(u)+k(1-x(u)) r(x(u)) \\
& \left.-\frac{1}{2}\left(\frac{a-b}{\sigma}\right)^{2}(1-x(u))^{2} x(u)^{2} r^{\prime}(x(u))\right\} d u \\
& +\int_{0}^{t}\left(\frac{a-b}{\sigma}\right)(1-x(u)) x(u) r(x(u)) d \nu(u)
\end{aligned}
$$


Since $r(x)$ is a solution of (5.3), using it in (5.23) gives

$$
\begin{aligned}
z(x(t))= & z\left(x_{0}\right)-\int_{0}^{t}\left\{a x(u)+b(1-x(u))-\frac{1}{2} \sigma^{2}\right\} d u \\
& +\int_{0}^{t}\left(\frac{a-b}{\sigma}\right)(1-x(u)) x(u) r(x(u)) d \nu(u)
\end{aligned}
$$

Consider a selling time $\tau$ for which $\tau \leq T(y)$ for some $y$ in $\left[0, x_{0}\right]$. Since $E[T(y)]<$ $\infty, E[\tau]<\infty$. Now (5.24) holds with $t$ replaced by $\tau$. Doing this and taking expectations gives

$$
E[z(x(\tau))]=z\left(x_{0}\right)-E\left[\int_{0}^{\tau}\left\{a x(u)+b\left(1-x(u)-\frac{1}{2} \sigma^{2}\right\} d u\right] .\right.
$$

The expected value of the stochastic integral is zero since its integrand is bounded and the expected value of it's upper limit is finite. Now

$$
\begin{aligned}
& E\left[\int_{0}^{\tau}\left\{a x(u)+b(1-x(u))-\frac{1}{2} \sigma^{2}\right\} d u\right] \\
= & E\left[z\left(x_{0}\right)-z(x(\tau))\right]=E\left[\int_{x(\tau)}^{x_{0}} r(z) d z\right] \leq \int_{y}^{x_{0}} r(z) d z .
\end{aligned}
$$

The last inequality holds since, $\mathrm{r}(\mathrm{z})$ is positive, and the definition of $\mathrm{T}(\mathrm{y})$ gives that $\tau \leq T(y)$ implies $x(\tau) \geq y$.

Using $T(y)$ for $\tau$ in (5.25) gives

$$
\begin{aligned}
& E\left[\int_{0}^{T(y)}\left\{a x(u)+b(1-x(u))-\frac{1}{2} \sigma^{2}\right\} d u\right] \\
= & E\left[z\left(x_{0}\right)-z(x(T(y))]=z\left(x_{0}\right)-z(y)=\int_{y}^{x_{0}} r(z) d z .\right.
\end{aligned}
$$

From (5.26) and (5.27) we see that selling at $T(y)$ is at least as good as selling at any time before it. It also follows that, as $y$ decreases selling at $T(y)$ increases the return. Thus selling should not occur before any $T(y)$ with $y>0$. Since as $y$ decreases to zero, $T(y)$ increases to $+\infty$, selling should not occur.

6. Some Computations and Conclusions. The results of a few computations are given in the table below. 


\begin{tabular}{|c|c|c|c|c|c|c|}
\hline$a$ & $-b$ & $c$ & $k$ & $\sigma$ & $r(x)+$ & $x^{*}$ \\
\hline .1 & .1 & 6 & 6 & .3 & no & .722 \\
\hline .1 & .1 & 6 & 12 & .3 & no & .717 \\
\hline .1 & .1 & 6 & 15 & .3 & no & .705 \\
\hline .1 & .1 & 6 & 16 & .3 & yes & \\
\hline .1 & .2 & 6 & 6 & .3 & no & .814 \\
\hline .1 & .2 & 6 & 18 & .3 & no & .810 \\
\hline .1 & .2 & 6 & 26 & .3 & no & .788 \\
\hline .1 & .2 & 6 & 27 & .3 & yes & \\
\hline 18 & .1 & 180 & 2 & .3 & yes & \\
\hline 14 & .1 & 180 & 2 & .3 & yes & \\
\hline 12 & .1 & 180 & 2 & .3 & no & .00088 \\
\hline 1 & .1 & 180 & 2 & .3 & no & .128 \\
\hline
\end{tabular}

In the case given in the first row of the table, the growth rates a and $-b$ are both .1 or $10 \%$ per year, the mean times spent in each these states $1 / c$ and $1 / k$ are both $1 / 6$ year or two months, the value .3 for $\sigma$ is one often obtained in estimation of stock price variances. The first three rows indicate how the conditional probability $x^{*}$ at which the stock should be sold changes as the mean time spent in state $b$ decreases. When this mean time reaches 1/16 year, or lower, the stock should be held. It seems very surprising, at least to the author, that even when the growth and decline rates are the same, that it is necessary that the mean time of decline be much less than the mean time of growth for holding the stock to be optimal. The next four rows illustrate a similar situation when the rate of decline is $20 \%$.

Stock analysts often advise their clients to hold their stocks because, the stocks growth is so rapid for such short periods, that they may miss the growth period if they sell and try to reinvest. The case in the last four rows is this type of stock. It has a large growth for a period with a mean of about two days and a slow decline for a period with mean six months. For the first two larger growth rates the stock should be held. For the next two smaller growth rates the stock should be sold,but only at very small values of the conditional probability.

The calculations above indicate that the "buy and hold" strategy is optimal only in very favorable circumstances. In row three, the expected decline during a period of decline, is only $6 / 15$ the expected growth during a period of growth, and yet the stock should be sold. In row seven, the expected decline during a period of decline, is only $6 / 13$ the expected growth during a period of growth, and yet the stock should be sold. In row eleven, the expected decline during a period of decline, is only 12/90 the expected growth during a period of growth, and yet the stock should be sold. 
Do the conclusions for this model have any relevance for real stocks? Certainly the behavior of a real stock's price is much more complicated than that of this model. Would fitting this model to data for a real stock, and using the results for the model give "good enough" results?

\section{REFERENCES}

[1] A. Bensoussan and J. L. Lions, Applications of Variational Inequalities in Stochastic Control, North Holland, 1982.

[2] Blanchet-Scallet, Diop, Gibson, Talay, Tanre, Technical Analysis Compared to Mathematical Models under Misspecification, NCCR-FINRISK Working Paper.

[3] Blanchet-Scallet, Diop, Gibson, Talay, Tanre, Technical Analysis Techniques Versus Mathematical Models: Boundaries of Their Validity Domains, In: Monte Carlo and Quasi Monte Carlo Methods 2004, H. Neiderreiter and D. Talay, Editors, Springer 2005.

[4] M. H. A. Davis and S. I. Markus, An Introduction to Nonlinear Filtering, In: Stochastic Systems: The Mathematics of Filtering and Identification and Applications, M. Hazwinkle and J. C. Willems, Editors, Riedel 1981.

[5] I. I. Ginman and A. V. Skoroknod, Stochastic Differential Equations, Springer-Verlag 1972.

[6] R. S. Lipster And A. N. Shiryayev, Statistics of Random Processes 1, General Theory, Springer-Verlag, 1977.

[7] R. Rishel and K. Helmes, A Variational Inequality Sufficient Condition for Optimal Stopping with Application to an Optimal Stock Selling Problem, SIAM Journal on Control and Optimization, to appear.

[8] A. N. Shiryayev, Optimal Stopping Rules, Springer-Verlag 1978.

[9] Q. Zhang, Stock Trading: An Optimal Selling Rule, SIAM Journal on Control and Optimization. Vol 40, 2001. 